\title{
Knowledge, Attitudes and Practice regarding personal cervical cancer screening among Clinical Staff Working in Obstetrics and Gynaecology at Two Tertiary Institutions in Jamaica \\ L Campbell $^{1}$ G Reynolds ${ }^{2}$, R Stevens ${ }^{1}$
}

\begin{abstract}
Objectives: The objective of the study was to evaluate knowledge, attitudes and practices with regards to cervical cancer screening among obstetric clinical staff.

Methods: A cross sectional study was carried out using questionnaires administered to female nurses and doctors employed to Obstetrics and Gynaecology at the University Hospital of the West Indies, Jamaica as well as the Victoria Jubilee Hospital. The sample comprised of 121 clinical staff members. Extracted data was analyzed using STATA 12® statistical software.

Results: The mean age of participants was 34.62 years. $77.8 \%$ thought that cervical screening should be done yearly. Only $50.41 \%$ considered themselves at risk of developing cervical cancer. $82.64 \%$ of participants had previously been screened. The most commonly stated deterrent to screening was a lack of time to attend clinics. Conclusion: While compliance with the practice of cervical screening is comparably favourable to other developing nations there is still need for education among healthcare workers particularly as it relates to risk factors and current international screening recommendations. Implementation of staff screening clinics may prove useful in improving compliance in this busy population.
\end{abstract}

Keywords: Attitudes and practices, cervical cancer, knowledge, Pap, Pap smear

From: ${ }^{1}$ Department of Obstetrics and Gynecology, Victoria Jubilee Hospital, North Street, Kingston,Jamaica, ${ }^{2}$ Faculty of Medical Sciences, University of the West Indies, Mona, Kingston 7, Jamaica.

Correspondence: Dr L Campbell, Department of Obstetrics and Gynaecology, The University of the West Indies, Kingston 7, Jamaica, West Indies. E-mail: drleroycampbell@gmail.com

\section{INTRODUCTION}


Cervical cancer remains the most common gynaecologic cancer in Jamaica and is second, only to breast cancer, in terms of malignancies afflicting Jamaican women (1). The implementation of organized national screening programs in countries such as the USA and the UK has led to dramatic falls in the cervical cancer rates (2) (3). In Jamaica the programme is not as well organized and screening is primarily caregiver driven. Not surprisingly our statistics remain comparatively poor. The incidence of cervical cancer is more than 4 times greater, in Jamaica than in the United States with the mortality rate locally being almost 6 times greater than rates in the United States (4).

Internationally recognized bodies such as the American Congress of Obstetrics and Gynaecology (ACOG), the U.S. Preventive Services Task Force and the American Cancer Society currently recommend that screening with cytology be performed every 3 years commencing at 21 years of age (5).

Much of the evidence reflecting knowledge attitudes and personal practices among clinical staff in developing nations has not been very favourable. Darj et al, in a cross sectional investigation of the nursing population in Tanzania made the observation that most nurses (84.6\%) had never had a Pap smear examination (6). Similarly another study conducted in Uganda found that $81 \%$ of health professionals had never been screened (7). In a Nigerian study only $14.1 \%$ of participants reported ever having had a Pap test (8). Only $13 \%$ of health care providers in one study conducted in the Democratic People's Republic of Korea (DPRK) had ever had a cervical cytology smear (9).

While these figures are quite alarming these findings may not necessarily translate to the Jamaican population. A local investigation was therefore considered necessary to evaluate the 
knowledge, attitudes and personal practices of our local clinicians as it relates to cervical cancer screening.

\section{METHODS}

A cross sectional study was done. Formal, written consent was obtained from each participant. Questionnaires were administered to female nurses and doctors employed to Obstetrics and Gynaecology at the University Hospital of the West Indies Jamaica as well as the Victoria Jubilee Hospital. Calculation of sample size was done using the Raosoft online calculator (10). The study was approved by the University of the West Indies ethics committee. Data analysis was done using STATA $12 \circledast$ statistical software.

\section{RESULTS}

A total of 121 clinical staff members participated in the study. As expected, from the relative distribution of staff at the institutions, the sample was comprised predominantly of nursing staff accounting for $87 \%$ as compared to Doctors (13\%). The mean age of participants was $34.62,95 \%$ CI $[33.2,36.04]$ years with a minimum age of 21 (one subject) and a maximum age of 52 . The majority (57.85\%) of the subjects had children. Among these the distribution was as follows: 
Table 1. Participants by number of children

\begin{tabular}{ll}
\hline Number of children & Participants(N=63) \\
\hline 1 & $60.32 \%, 95 \%$ CI $[48.24,72.4]$ \\
2 & $25.4 \%, 95 \%$ CI $[14.65,36.15]$ \\
3 & $12.70 \%, 95 \%$ CI $[4.48,20.92]$ \\
4 & $1.59 \%, 95 \%$ CI $[-1.5 \%, 4.68]$ \\
\hline
\end{tabular}

\section{Knowledge}

Table 2: Where does cervical cancer rank among cancers in Jamaican women in terms of frequency $(\mathrm{N}=118)$ ?

Most common
Second most common
Third most common
Rare
Don't know

Most common

Third most common

Don't know

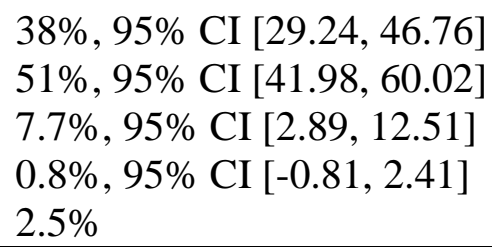

$38 \%, 95 \%$ CI $[29.24,46.76]$

$51 \%, 95 \%$ CI $[41.98,60.02]$

$7.7 \%, 95 \%$ CI $[2.89,12.51]$

$0.8 \%, 95 \%$ CI $[-0.81,2.41]$

$2.5 \%$

Table 3: What is the purpose of the pap smear $(\mathrm{N}=105)$ ?

To check for cervical cancer

To check for precancerous lesions of the cervix

To check for inflammation of the cervix
$31 \%, 95 \%$ CI $[22.15,39.85]$

$68 \%, 95 \%$ CI [59.08, 76.92]

$1 \%, 95 \%$ CI $[-0.9,2.9]$

Table 4: How often should women be screened with a pap smear $(\mathrm{N}=117)$ ?

At least every 5 years starting at age 21

At least once every year starting at age 21

At least once every 3 years starting at age 21

At least once every year after age 50
$1.7 \%, 95 \%$ CI $[-0.64,4.04]$

$77.8 \%, 95 \%$ CI $[70.27,85.33]$

$18.8 \%, 95 \%$ CI $[11.72,25.88]$

$1.7 \%, 95 \%$ CI $[-0.64,4.04]$ 
The questionnaire listed six of the known risk factors for cervical cancer. Only two respondents were able to identify all six. Table 5 demonstrates the number of risk factors identified and the proportion of respondents.

Table 5: Risk factors

\begin{tabular}{lrlr}
\hline $\begin{array}{l}\text { Number of } \\
\text { identified }\end{array}$ & risk factors & $\begin{array}{l}\text { Number of } \\
\text { (percent } \\
\text { respondents) }\end{array}$ of & \\
1 & & $25(20.7 \%)$ & \\
2 & & $18(14.9 \%)$ & \\
3 & & $28(23.1 \%)$ & \\
4 & & $31(25.6 \%)$ & \\
5 & & $17(14.0 \%)$ & \\
6 & & $2(1.7 \%)$ & \\
\hline
\end{tabular}

\section{Attitudes}

Table 6. Do you think that you are at risk of developing cervical cancer $(\mathrm{N}=117)$ ?

Yes

No.

Don't know
$50.41 \%, 95 \%$ CI [41.35, 59.47]

$31.40 \%, 95 \%$ CI $[22.99,39.81]$

$14.88 \%, 95 \%$ CI $[8.43,21.33]$ 


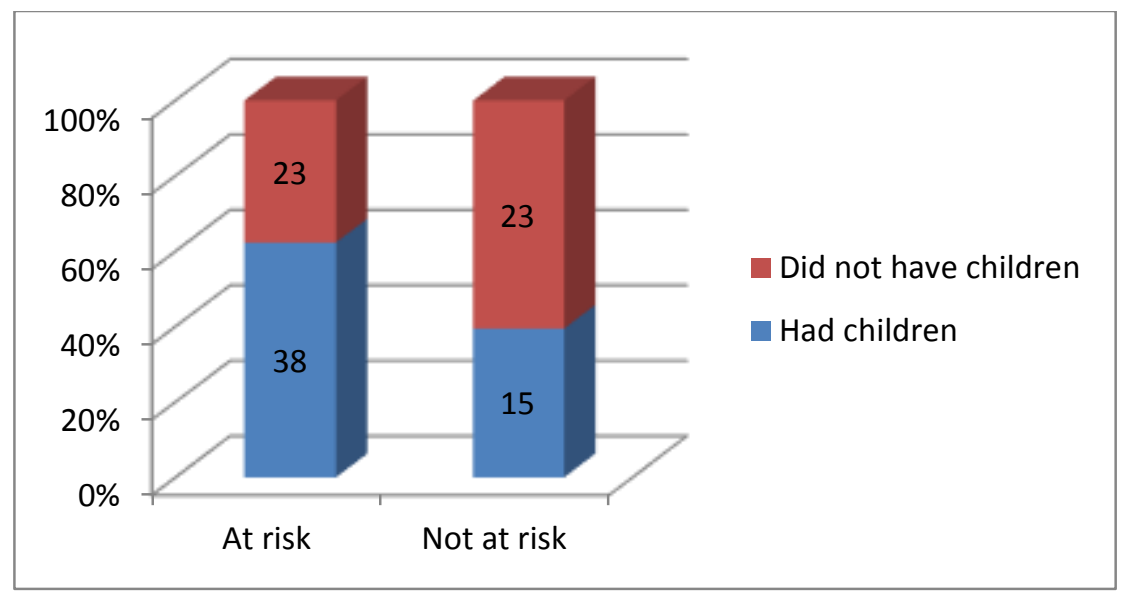

Fig. 1. Association between parity and opinion of personal susceptibility.

Subjects who considered themselves to be at risk of cervical cancer were more likely to have had children than those who did not consider themselves to be at risk (odds ratio 2.53, 95\% CI[1.1031, $5.8180]$.

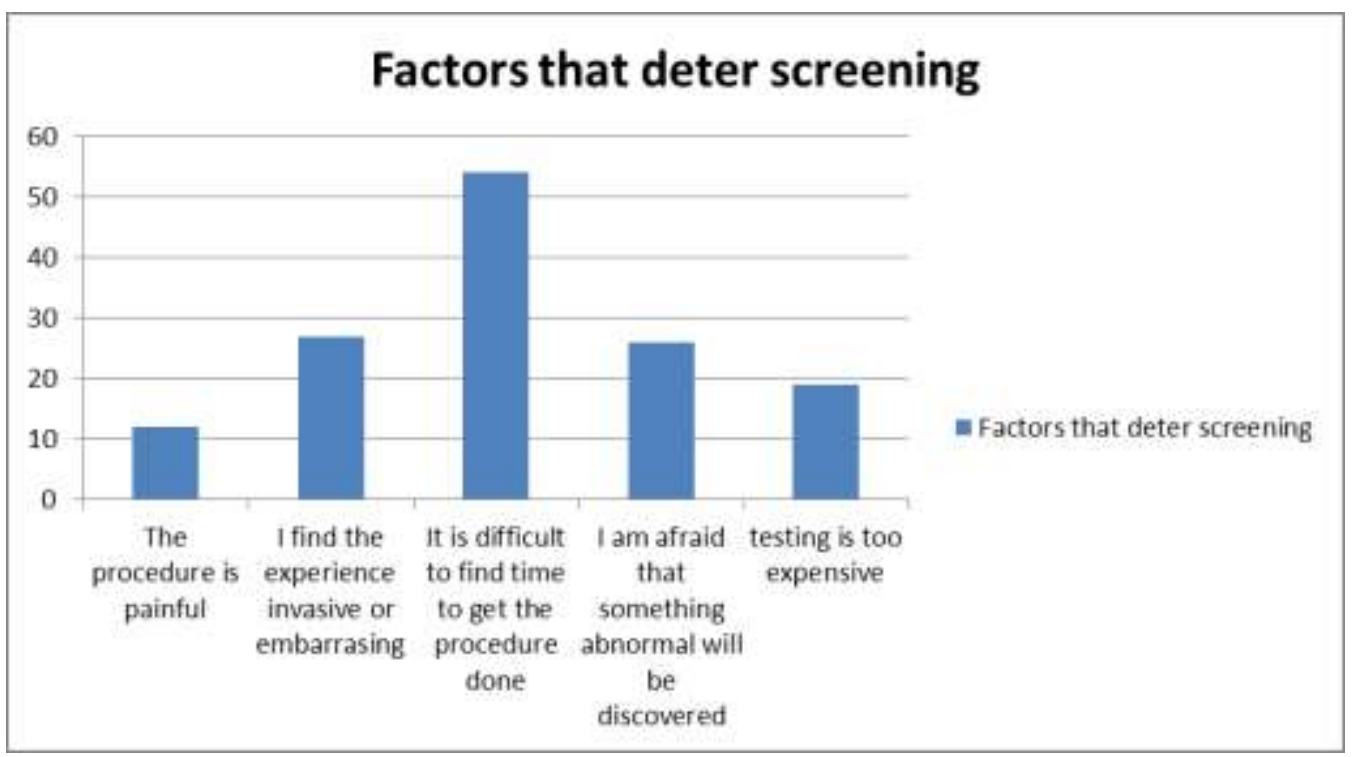

Fig 2: Deterrents to regular screening. 


\section{Practice}

Table 7. Have you ever had a pap smear $(\mathrm{N}=121)$ ?

Yes

$82.64 \%, 95 \%$ CI $[75.89,89.39]$

No

$17.36 \%, 95 \%$ CI $[10.61,24.11]$

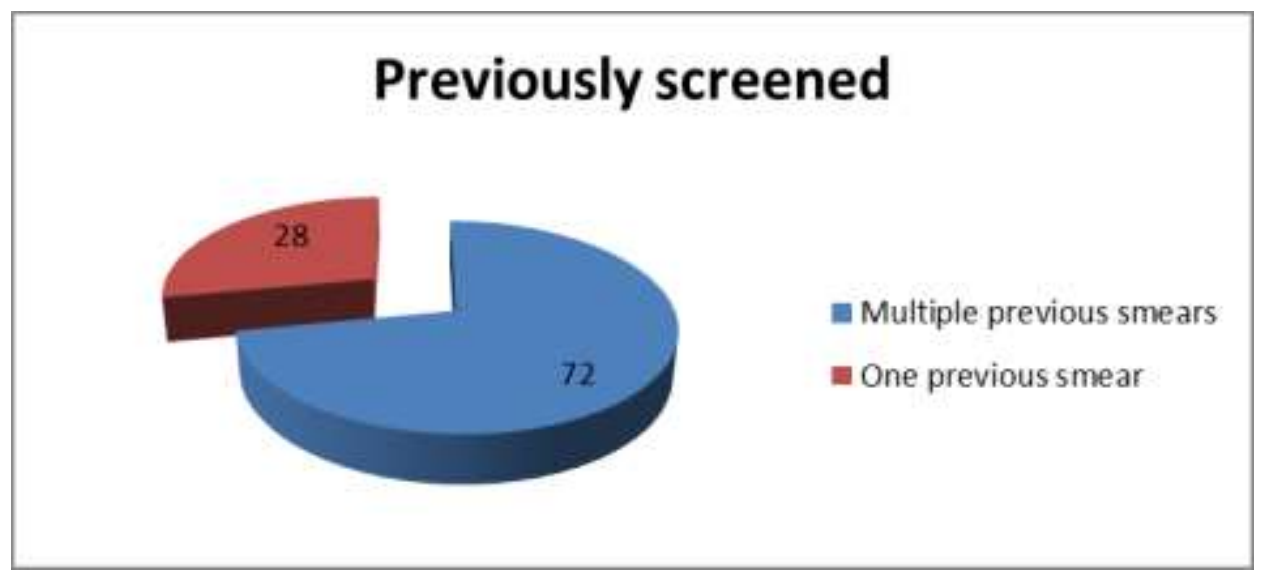

Fig. 3: Previously screened patients.

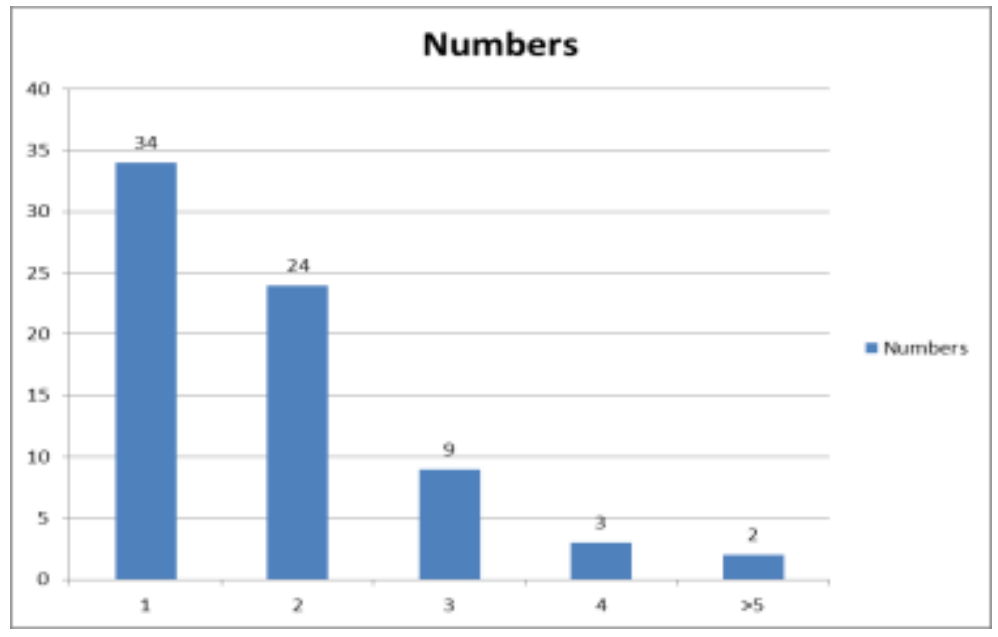

Fig. 4: Interval (in Years) between pap smears. 


\section{DISCUSSION}

The majority of respondents in the study correctly identified cervical cancer as a major public health problem. A similar observation was made in a study by Mutyaba et al (7). This was not a surprising observation as cervical cancer patients represent a significant proportion of gynaecological ward admissions. The absence of a formal local screening programme means that it is not uncommon for women to be diagnosed in the advanced stages of cervical cancer when, bleeding or constitutional symptoms prompt presentation for care. Complications such as uraemia from obstructive uropathy, recurrent vaginal haemorrhage and intractable pain from bone metastases can make a significant impression on caregivers. This may explain the observation that although only half of the respondents considered themselves to be at risk of developing cervical cancer over $80 \%$ had previously been screened.

Human papilloma virus (HPV) is central to the development of cervical cancer and can be detected in $99.7 \%$ of cases (11). As a result of this aetiology the epidemiology of cervical cancer closely resembles that of a sexually transmitted disease. Many of the risk factors for cervical cancer are the same as those for sexually transmitted infection, including early age at onset of sexual activity, multiple pregnancies, and multiple sexual partners (12). While not fully understood it has been observed that the relative risk of cervical cancer is increased in current users of oral contraceptives and declines after use ceases (13).

In our study all the participants were able to identify at least one risk factor for cervical cancer. Only 2 of the 121 respondents to this question were able to identify all six of the stated risk factors. This suggests that while there is a basic knowledge of the risk factors associated with cervical cancer there is still room for health education on this subject.

The Pap test aims to identify abnormal cells sampled from the transformation zone (14). Most subjects in the study correctly identified that the purpose of the pap smear was to check for precancerous lesions of the cervix (Table 3). A third of the participants believed that the pap smear 
was used to check for cervical cancer, while one participant was under the impression that the purpose of the test was to check for inflammation of the cervix.

The diagnosis of invasive cancer is based on histologic assessment of a cervical biopsy specimen. The Pap smear is not an effective tool for the diagnosis of cervical cancer and it is not uncommon for smears to misdiagnose overt malignancy. While inflammatory changes may be a commonly reported abnormality on cytology the purpose of the test is not to diagnose cervical inflammation.

While current local practices are widely caregiver driven screening practices have trended towards consistency with internationally accepted recommendations. Screening with cervical smears is recommended three yearly commencing at age 21 years regardless of sexual history (7). Previous guidelines recommended yearly screening with screening commencing at 18 years of age or after coitarche; whichever came first(15). Three quarters of the clinical staff sampled thought that cervical cancer screening was to be performed every year commencing at the age of 21 years (Table 4). This finding reflects a lack of up to date knowledge of the current international screening recommendations. Interestingly, however, in spite of the fact that over $75 \%$ of participants thought that screening was to be done yearly, only $47.2 \%$ of the subjects who had multiple previous pap smears (Figure 3) reported an interval of 1 year between smears (Figure 4).

In 2011, a publication from the U.S. Preventive Services Task Force suggested that twice as many colposcopies would be performed if screening were performed annually compared with screening every three years, with only a slightly greater reduction in cancer risk (16). An interval of one year is associated $93.5 \%$ reduction compared to $90.8 \%$ when an interval of 3 years was used (17). Dissemination of this information is critical in our local, developing, economy; as compliance with newer recommendations would likely yield significant fiscal benefit. Although the vast majority recognized that cervical cancer was an important public health problem in 
Jamaica, the feeling of personal susceptibility was not, nearly, as universal. Only half of the participants thought that they were at risk of developing cervical cancer (Table 6) a finding similar to those described by Mutyaba et al (7). The reason for this observation is not clear. Perhaps a lack of knowledge of the risk factors for the disease might have led to an erroneous assumption by the participant that she was not at risk. Conversely the participant may have had a good knowledge of the risk factors but did not feel that she displayed any of these factors.

One observation in the data is that patients who considered themselves to be at risk of developing cervical cancer were more likely to have had children than participants who thought they were not at risk (Odds ratio $2.5,95 \%$ CI [1.1031 to 5.8180]) (figure 1). One possible explanation is that parous respondents may have been exposed to additional health promotion during antenatal visits.

The most frequently stated deterrent to screening was a lack of time (Figure 2). This observation is not surprising as nurses and doctors, by nature of their profession, are very busy people. It is not uncommon for clinical staff in Public hospitals in Jamaica to work very long hours to compensate for staff shortages. Development of hospital based staff oriented clinics may make screening more convenient and thus improve compliance.

$80 \%$ of respondents had been screened prior. This, though not ideal, compared favourably to the findings of previously published articles $(6,7,8,9)$.

\section{CONCLUSION}

While compliance with the practice of cervical screening is comparably favourable to other developing nations there is still need for education among healthcare workers particularly as it 
relates to risk factors and current international screening recommendations. Intervention is necessary to secure the welfare of our work force; lest in the course of saving others they neglect themselves.

\section{REFERENCES}

1. Gibson TN, Hanchard B, Waugh N, McNaughton D. Age-specific incidence of cancer in Kingston and St. Andrew, Jamaica, 2003-2007. West Indian Med J 2010; 59: 456-64. 
2. Gustafsson L, Ponten J, Zack M, Adam Ho. International incidence of invasive cervical cancer after introduction of cytological screening. Cancer Causes Control. 1997; 8: $755-63$.

3. Cuzick J, Boyle P.Trends in cervical cancer mortality. Cancer Surv 1988; 7: 417-39.

4. Lewis, MJ. A Situational Analysis of Cervical Cancer in Latin America. Washington DC: Panamerican Health Organization. 2004.

5. ACOG. New Cervical Cancer Screening Recommendations from the U.S. Preventive Services Task Force and the American Cancer Society/American Society for Colposcopy and Cervical Pathology/American Society for Clinical Pathology. The American Congress of Obstetricians and Gynecologists. [Online] March 14, 2012. [Cited: August 1, 2012.] Available from: http://www.acog.org.

6. Urasa M, Darj E. Knowledge of cervical cancer and screening practices of nurses at a regional hospital in Tanzania. Afr Health Sci 2011; 11: 48-57.

7. Mutyaba T, Mmiro FA, Weiderpass E. Knowledge, attitudes and practices on cervical cancer screening among the medical workers of Mulago Hospital, Uganda, BMC Med Educ 2006; 6: 13.

8. Gharoro EP, Ikeanyi EN. An appraisal of the level of awareness and utilization of the Pap smear as a cervical cancer screening test among female health workers in a tertiary health institution. Int J Gynecol Cancer. May-Jun 2006; 16: 1063-8.

9. Tran NT, Taylor R, Pyo HS. Knowledge, attitude and practice (KAP) concerning cervical cancer and screening among rural and urban female healthcare practitioners in the Democratic People's Republic of Korea. Asian Pac J Cancer Prev 2011; 12: 3023-8. 
10. Raosoft, Inc. Raosoft Sample size calculator. http://www.raosoft.com/. [Online] [Cited: September 23, 2012.]

11. Walboomers JM, Jacobs MV, Manos MM, Bosch FX, Kummer JA, Shah KV. Human papillomavirus is a necessary cause of invasive cervical cancer worldwide. J Pathol 1999; 189: 12 .

12. Hoskins, William J, Perez, Carlos A. and Young, Robert, Barakat, Markman, Randall. Principles and Practice of Gynecologic Oncology, 4th Edition. s.l. : Lippincott Williams and Wilkins, 2005.

13. International Collaboration of Epidemiological Studies of Cervical Cancer, Appleby P, Beral V, Berrington de González A, Colin D, Franceschi S, Goodhill A et al. Cervical cancer and hormonal contraceptives: collaborative reanalysis of individual data for 16,573 women with cervical cancer and 35,509 women without cervical cancer from 24 epidemiological studies. Lancet 2007; 10; 370: 1609-21.

14. Feldman S, Sirovich B, Goodman A. Screening for cervical cancer: Rationale and recommendations. UpToDate, Inc. 2012

15. Barakat, Bevers, Gershenson, Hoskins. Handbook of Gynaecologic Oncology. Second Edition. London : Martin Dunitz, 2000.

16. Kulasingam SL, Havrilesky L, Ghebre R, Myers ER. Screening for Cervical Cancer: A decision analysis for the U.S. Preventative Services Task Force. Rockville, MD: Agency for Healthcare Research and Quality, 2011 Report No.: 11-05157-EF-1

17. IARC Working Group on evaluation of cervical cancer screening programmes. Screening for squamous cervical cancer: duration of low risk after negative results of cervical 
cytology and its implication for screening policies. Br Med J (Clin Res Ed) 1986; 13: 65964. 